\title{
How soon would the next mega-earthquake occur in Japan?
}

\author{
Alexey Lyubushin \\ Institute of Physics of the Earth, Russian Academy of Sciences, Moscow, Russia; lyubushin@yandex.ru
}

Received 12 May 2013; revised 12 July 2013; accepted 19 July 2013

Copyright (c) 2013 Alexey Lyubushin. This is an open access article distributed under the Creative Commons Attribution License, which permits unrestricted use, distribution, and reproduction in any medium, provided the original work is properly cited.

\begin{abstract}
The problem of seismic danger estimate in Japan after Tohoku mega-earthquake 11 March of 2011 is considered. The estimates are based on processing low-frequency seismic noise waveforms from broadband network F-net. A new method of dynamic estimate of seismic danger is used for this problem. The method is based on calculating multi-fractal properties and minimum entropy of squared orthogonal wavelet coefficients for seismic noise. The analysis of the data using notion of "spots of seismic danger" shows that the seismic danger in Japan remains at high level after 2011. 03. 11 within north-east part of Philippine plate-at the region of Nankai Though which traditionally is regarded as the place of strongest earthquakes. It is well known that estimate of time moment of future shock is the most difficult problem in earthquake prediction. In this paper we try to find some peculiarities of the seismic noise data which could extract future danger time interval by analogy with the behavior before Tohoku earthquake. Two possible precursors of this type were found. They are the results of estimates within 1-year moving time window: based on correlation between 2 mean multi-fractal parameters of the noise and based on cluster analysis of annual clouds of 4 mean noise parameters. Both peculiarities of the noise data extract time interval 2013-2014 as the danger.
\end{abstract}

Keywords: Seismic Noise; Multi-Fractal Analysis; Wavelet-Based Minimum Normalized Entropy; Cluster Analysis; Earthquake Prediction; Dynamic Estimate of Seismic Danger

\section{INTRODUCTION}

The problem of predicting strongest earthquakes in
Japan at the region of Nankai Trough is a traditional large problem for seismologists in Japan [1,2]. In [1] the probability of earthquake with magnitude more than 8.5 at Tokai-Nankai zone, the region where Philippine Sea plate is approaching Central Japan, was estimated as 0.35 - 0.45 “for a ten-year period following the year 2000". In [3] the seismic danger for Japan was estimated immediately after Tohoku earthquake based on the analysis of GPS data and the conclusion was that "estimates ... suggest the need to consider the potential for a future large earthquake just south of this event.” In the paper [4] the problem why the Tohoku earthquake was a surprise for scientific community is discussed. One of the conclusions in [4] is "A magnitude 9 earthquake off Tohoku should not have been a surprise". This conclusion was made by retrospective analysis of seismic catalogs. Nevertheless the Tohoku event was a great surprise for all traditional methods of earthquake prediction. Other conclusion in [4] is that even magnitude 10 is quite possible for Japan Trench.

Broadband seismic networks provide other type of information which could be used for earthquake prediction as well. Low-frequency microseismic oscillations and their correlation with the processes occurring in the hydrosphere and atmosphere of the Earth were investigated in [5-7].

In papers [8-12] an analysis of the multi-fractal parameters of low-frequency seismic noise from the network F-net provided a hypothesis that Japanese Islands were approaching a large seismic catastrophe, the signature of which was a statistically significant decrease in the support width of the multi-fractal singularity spectrum. A cluster analysis of background parameters led us to conclude that in the middle of 2010 the islands of Japan entered a critically dangerous developmental phase of seismic process [11] (the paper [11] was submitted at April of 2010). The prediction of the catastrophe, first in terms of approximate magnitude (middle of 2008) and then in terms of approximate time (middle of 2010), was documented in advance in a series of papers and in pro- 
ceedings at international conferences [5-11].

This paper is an immediate continuation of the paper [13] where a new approach for dynamic estimate of seismic danger based on investigation of continuous records of seismic noise was elaborated. Different aspects of the method were published in $[14,15]$ as well. At the current paper the main purpose is an effort to find estimates of the time of possible new Japanese mega-earthquake in Tokai-Nankai zone.

\section{DATA}

For the analysis a vertical broadband seismic oscillations components with 1-second sampling time step (LHZ-records) from the broad-band seismic network F-net stations in Japan were downloaded from internet address http://www.fnet.bosai.go.jp starting from the beginning of 1997 up to 30 of April 2013. We considered the stations which are located northward from $30^{\circ} \mathrm{N}$ and, thereby excluding the data from 6 solitary stations located on remote small islands. The locations of 78 stations (one new station was added at May 2011) which were chosen for analysis are indicated in Figure 1 with epicenters of 2 the strongest earthquakes which occurred during observations: near Hokkaido at 25 of September 2003 with magnitude 8.3 and Tohoku mega-earthquake at 11 of March 2011 with magnitude 9.0. In this paper the seismic data were analyzed after transforming them to sampling time step 1 minute by calculating mean values within adjacent time windows of the length $60 \mathrm{sec}$. Thus, the minimum period of seismic noise variations for the analysis equals 2 minutes.

\section{METHODS AND RESULTS}

\subsection{Multi-Fractal Singularity Spectrum Parameters}

Multifractal singularity spectrum $F(\alpha)$ [16] of the signal $x(t)$ is defined as a fractal dimensionality of time moments $t_{\alpha}$ which have the same value of Lipschitz-Holder exponent $h(t)=\lim \left(\ln \left(\mu_{t}^{(\delta)}\right) / \ln (\delta)\right)$, i.e. $h\left(t_{\alpha}\right)=\alpha$, where $\mu_{t}^{(\delta)}=\stackrel{\delta \rightarrow 0}{\max } x(s)-\min x(s)$, maximum and minimum values are taken for argument $t-\delta / 2 \leq s \leq t+\delta / 2$. The value $\mu_{t}^{(\delta)}$ is a measure of signal variability in the vicinity of time moment $t$. Practically the most convenient method for estimating singularity spectrum is a multifractal DFA-method [17] which is used here. The function $F(\alpha)$ could be characterized by following parameters: $\alpha_{\min }, \alpha_{\max }, \Delta \alpha=\alpha_{\max }-\alpha_{\min }$ and $\alpha^{*}$-an argument providing maximum to singularity spectra: $F\left(\alpha^{*}\right)=\max F(\alpha)$.

Parameter $\alpha^{*}$ could be called a generalized Hurst exponent and it gives the most typical value of Lipschitz-Holder exponent. Parameter $\Delta \alpha$, singularity

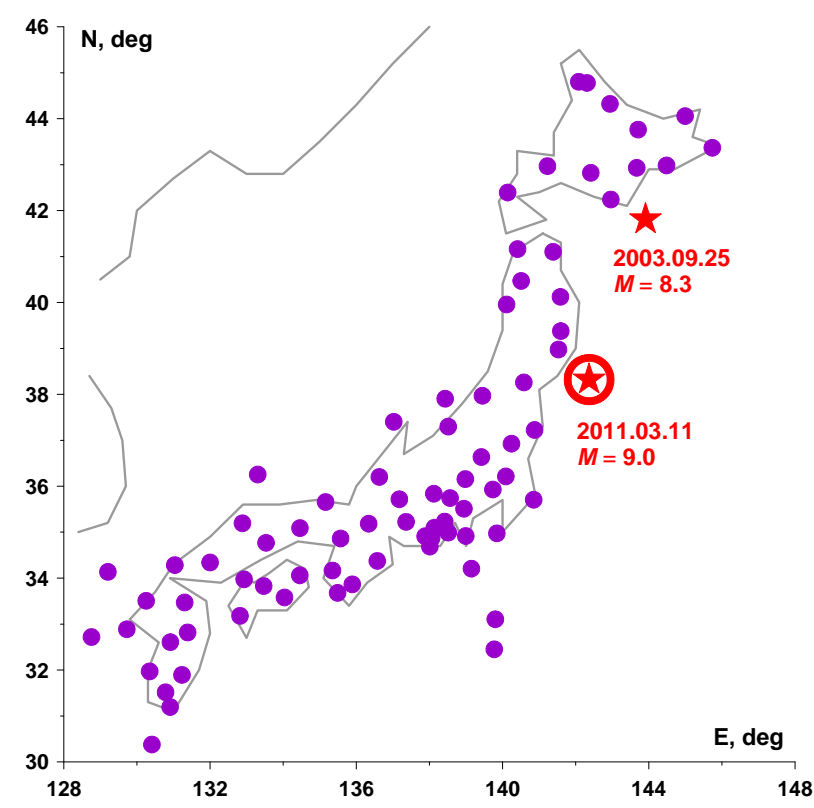

Figure 1. Positions of 78 seismic stations of the network F-net.

spectrum support width, could be regarded as a measure of variety of stochastic behavior. For removing scaledependent trends (which are mostly caused by tidal variations) in multi-fractal DFA-method of singularity spectrums estimates a local polynomials of the 8-th order were used.

\subsection{Wavelet-Based Minimum Normalized Entropy}

Let us consider finite piece of random signal $x(t)$, where $t=1, \ldots, N ; N$ is the number of samples. The normalized entropy $E n$ of the distribution of squared orthogonal wavelet coefficients is defined by the formula:

$$
E n=\sum_{k=1}^{N} p_{k} \ln \left(p_{k}\right) / \ln (N), p_{k}=c_{k}^{2} / \sum_{j=1}^{N} c_{j}^{2}
$$

According to definition (1) entropy $E n$ is normalized: $0 \leq E n \leq 1$. Here $c_{k}, k=1, \ldots, N$ are the orthogonal wavelet coefficients of some basis. We used 17 Daubechies orthogonal wavelets: ten ordinary bases with the minimum support length with one to ten vanishing moments and seven so called Daubechies symlets [18] with four to ten vanishing moments. For each basis, the normalized entropy of the distribution of squared coefficients was calculated by formula (1), and the basis rendering the minimum of (1) was determined. As the wavelets are the orthogonal transform, the sum of their squared coefficients is equal to the variance of the signal $x(t)$. Thus, quantity (1) describes the entropy of the oscillation energy distribution on various spatial and temporal scales. For seismic noise the parameter En was estimated within adjacent time windows of the length 1 day, after removing trend by polynomial of the 
8-th order.

\subsection{Averaged Maps of Seismic Noise Properties}

The seismic records from each stations after coming to 1 minute sampling time step were split into adjacent time fragments of the length 1 day (1440 samples) and for each fragment parameters $\Delta \alpha$ and $E n$ were calculated. Thus, time series of these 2 values with sampling time step 1 day were obtained from each of 78 seismic stations which are presented at the Figure 1.

Having the values of $\Delta \alpha$ and $E n$ from all seismic stations it is possible to create maps of spatial distribution of these seismic noise statistics. For this purpose let us consider the regular grid of the size $30 \times 30$ nodes covering the rectangular domain with latitudes between $30^{\circ} \mathrm{N}$ and $46^{\circ} \mathrm{N}$ and longitudes between $128^{\circ} \mathrm{E}$ and $148^{\circ} \mathrm{E}$ (see Figure 1). For each node of this grid the daily val-
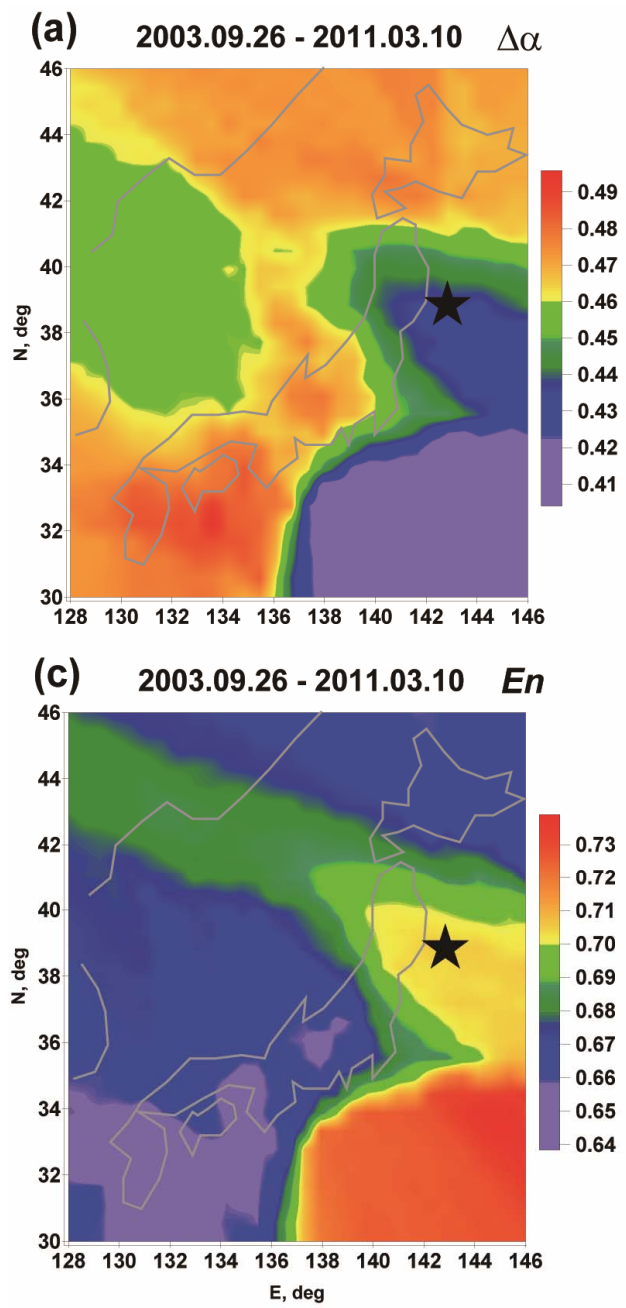

ues of $\Delta \alpha$ and $E n$ are corresponded which are calculated as median for the values of 5 nearest to the node seismic stations. This simple procedure provides the sequence of daily maps of parameters. The averaged maps are created by averaging daily maps for all days between and including 2 given dates. Taking into account that almost all stations of the F-net are placed at large Japanese islands these map in the ocean regions have the less significance than at islands of course. The used method of nearest neighbors provides a rather natural extrapolation of the used values into domains which have no points of observations.

Figure 2 presents such maps for 2 adjacent time intervals: from 2003. 09. 26 (immediately after earthquake near Hokkaido—see Figure 1) up to 2011. 03. 10 (just before the Tohoku mega-earthquake)—Figures 2(a), (c) and from 2011. 03. 14 (when the system F-net started to work normally after Tohoku shock) up to 2013. 04. 30Figures 2(b), (d).
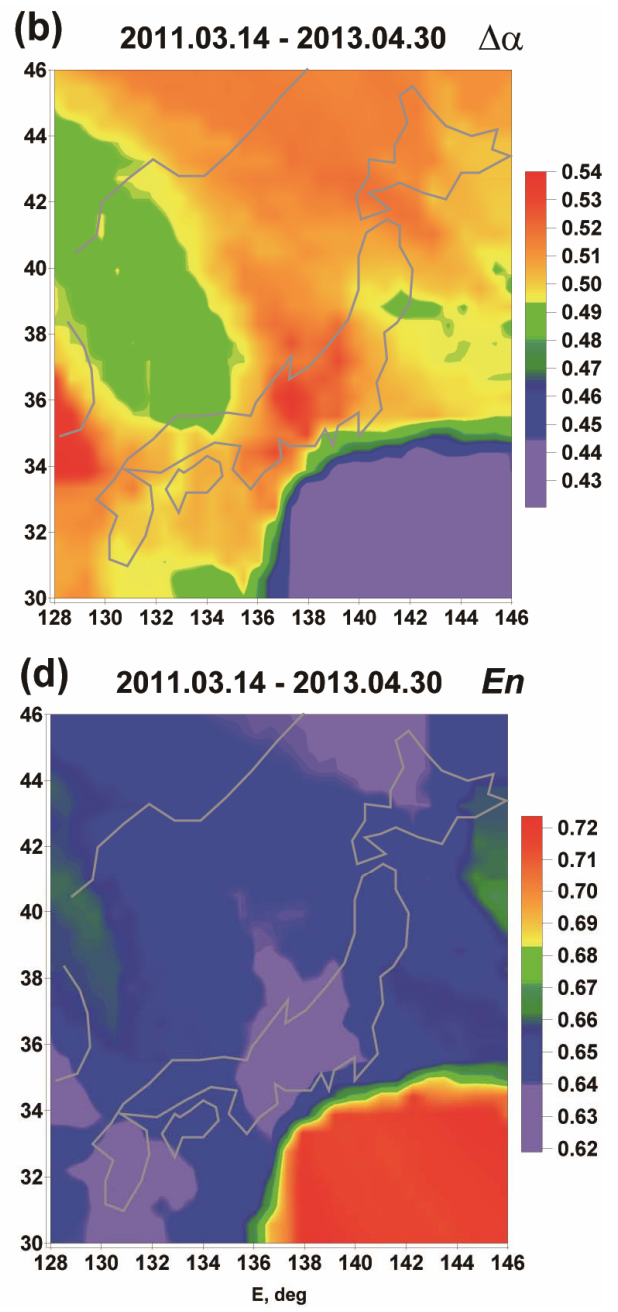

Figure 2. Averaged maps of multi-fractal singularity spectrums support width (a), (b) and minimum normalized entropy (c), (d) for 2 successive time intervals. Star indicates epicenter of earthquakes 11 of March 2011, $\mathrm{M}=9.0$ (a), (c). 
The main hypothesis of new method for dynamic estimate of seismic danger consists in the proposition that averaged maps of spatial distribution of seismic noise statistics $\Delta \alpha$ and $E n$ could extract the place of future catastrophe as the regions with relatively low values of $\Delta \alpha$ and relatively high values of En [13-15]. Let us call the regions extracted by low values of $\Delta \alpha$ and high values of $E n$ as "spots of seismic danger"-SSD. Figures 2(a), (c) illustrate the main hypothesis: the region of future Tohoku earthquake was a large SSD during time period from 2003. 09. 26 up to 2011. 03. 10. The analysis of seismic noise after 2011.03.14 extracts the region of Nankai Trough as remaining to be SSDFigures 2(b), (d). The fact that this Nankai Trough region was SSD before Tohoku earthquake and remains to be SSD after the event arises another hypothesis that during Tohoku earthquake only a half of accumulated tectonic energy was dropped and the next mega-earthquake is possible in the region which is in southern direction from the region of Tohoku earthquake. The same hypothesis arose after analyzing GPS data and was published in [3].

Figure 3 presents examples of 4 daily noise waveforms with different values of $\Delta \alpha$ and $E n$ : left-hand panels of graphics, Figures 3(a), (b), present noise waveforms with high value of $\Delta \alpha$ and low values of $E n$ whereas right-hand panels, Figures 3(c), (d), correspond to 2 noise waveforms with low values of $\Delta \alpha$ and high values of $E n$. The difference in waveforms peculiarities between Figures 3(a), (b) and Figures 3(c), (d), is rather evident: high values of $\Delta \alpha$ and low values of $E n$ occur because of existence of irregular high-amplitude spikes which are intermitted with intervals with stationary behavior. This is a typical multi-fractal: different types of stochastic behavior are observed. Low values of $\Delta \alpha$ correspond to much more stationary behavior: the noise structure is more simple and less multi-fractal.

A possible physical interpretation of ability of low values of $\Delta \alpha$ and high values of $E n$ extract seismically dangerous regions was given in [13-15]. It is the consequence of consolidation of small blocks of the Earth's crust into the large one before the strong earthquake. Consolidation follows that seismic noise does not include spikes which are connected with mutual movements of small blocks. The absence of irregular spikes in the noise follows the decreasing of $\Delta \alpha$ and increasing of entropy $E n$.

\subsection{Correlations between Generalized Hurst Exponent and Singularity Spectrum Support Width}

Figure 4(a) presents variations in the robust estimate [19] of squared correlation coefficient $\rho^{2}$ between increments of daily median values of multi-fractal param-
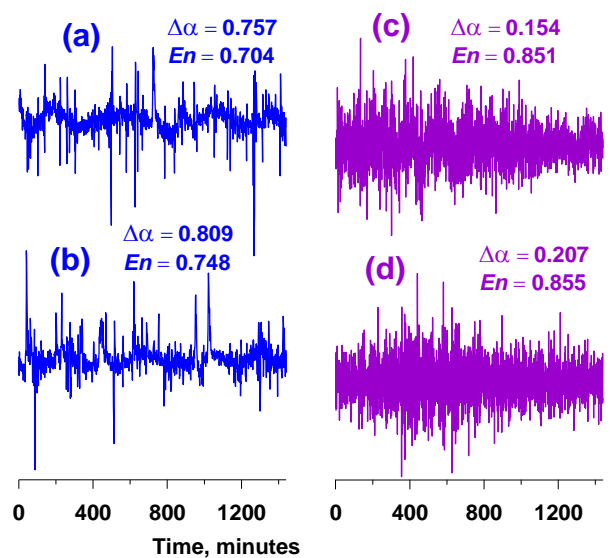

Figure 3. Two types of daily low-frequency seismic noise waveforms after removing tidal trends by polynomial of $8^{\text {th }}$ order: (a), (b) - with relatively large values of singularity spectrum support width $\Delta \alpha$ and high values of normalized entropy $E n$ and (c), (d) - with relatively low values of $\Delta \alpha$ and En.

eters $\alpha^{*}$ and $\Delta \alpha$ in a moving time window with a length of 365 days. Figure 4(a) is notable in that it displays two prominent anomalies in the behavior of the correlation coefficient before the Tohoku earthquake: sharp minima in 2002 and 2009. The first anomaly of 2002 occurred before the large earthquake of 2003. 09 . 25; therefore, it was logical to expect that the second sharp minimum of the correlation coefficient could also be a precursor to a future strong (and, possibly, even higher energy) event in the second half of 2010. From this dependence we could conclude [11,12] that, starting from the middle of 2010, a strong event with magnitude more than 8.3 should be expected on the islands of Japan. After 2011.03.11 the estimate of squared correlation coefficient forms a new sharp minimum with position of right-hand end of 1 year moving time window at the beginning of 2012. This fact provides a foundation to propose that the next mega-earthquake at the region of low values of $\Delta \alpha$ could occur within time interval 2013$2014[14,15]$.

Similar to the maps presented at the Figure 2 we can plot averaged maps of $\rho^{2}$. For this purpose let's estimate evolution of $\rho^{2}$ for each station within moving time window of the length 365 days. For each position of 1 -year moving time window we can plot a $\rho^{2}$-map by calculating median of $\rho^{2}$-values for 5 seismic stations which are nearest to each node of regular grid. The averaged maps are created by averaging maps corresponding to all 1-year time fragments which lay entirely between and including 2 given dates.

Such $\rho^{2}$-maps are presented at Figures 4(b), (c). It is interesting to notice that at Figure $4(\mathbf{b})$ the region of future Tohoku earthquake is extracted by relatively high values of $\rho^{2}$. For time period after Tohoku earthquake 
(b) $2003.09 .26-2011.03 .10$

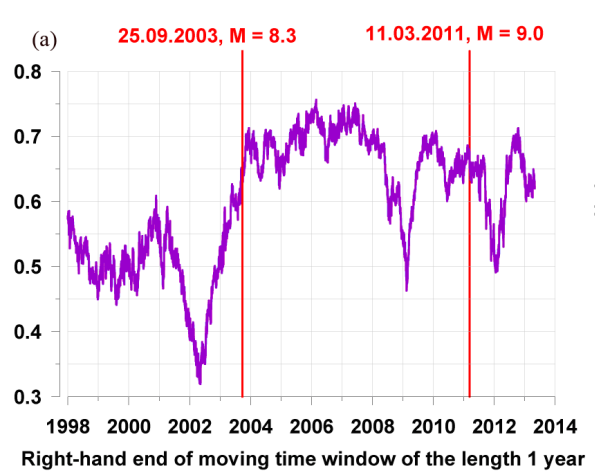

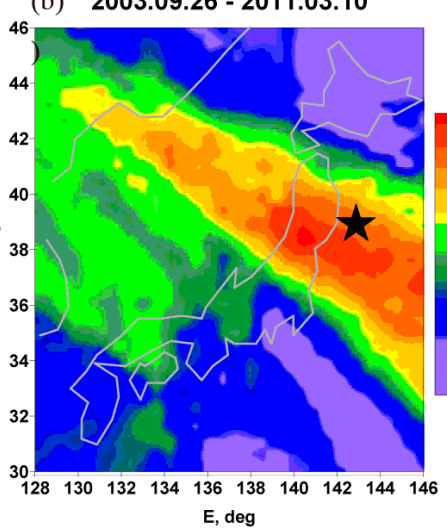

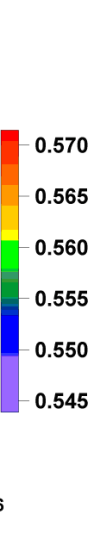

(c) 2011.03.14 - 2013.04.30

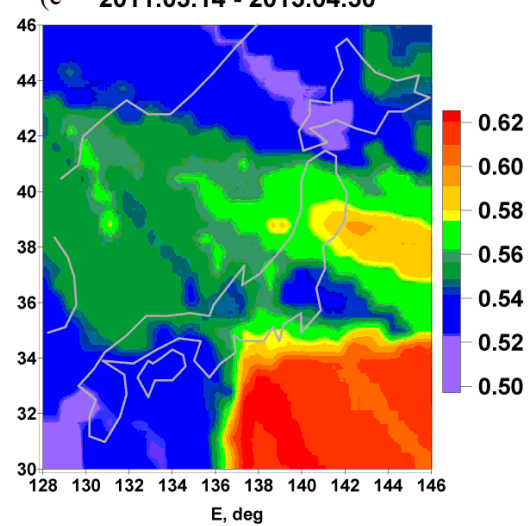

Figure 4. (a) - estimate of squared robust correlation coefficient between median values of multi-fractal singularity spectrums support width $\Delta \alpha$ and generalized Hurst exponent $\alpha^{*}$ within 1-year moving time window; (b, c) —averaged maps of squared correlation coefficient between $\Delta \alpha$ and $\alpha^{*}$ estimated within moving time window of the length 365 days for 2 adjacent time fragments; star indicates epicenter of earthquakes 11 of March 2011, $M=9.0$ (b).

the region of SSD according to Figures 2(b), (d) coincides with region of maximum values of $\rho^{2}$-Figure 4(c). Unlike situation with SSD, extracted by low values of $\Delta \alpha$ and high values of $E n$, such prognostic properties of $\rho^{2}$ have no possible physical interpretation now.

\subsection{Cluster Analysis within Moving Time Window}

The previous analysis of correlation $\rho^{2}$ has a purpose to find some peculiarities of the data which could help in estimating the time moment of the future earthquake what is the most difficult problem in earthquake prediction. This section of the paper is devoted to the same problem. But the method is based on cluster analysis.

Let's consider 4-dimensional (4D) vector $\vec{\xi}$ which consists of median values of 3 parameters of multi- fractal singularity spectrum $\Delta \alpha, \alpha^{*}, \alpha_{\min }$ and minimum normalized entropy $E n$ which were computed each day using information from all stations. The graphics of scalar components of the vector $\vec{\xi}$ are presented at the Figures 5(a)-(d).

Let us consider moving time window of the length $L=$ 365 days and let $\vec{\xi}^{(t)}$ be $4 \mathrm{D}$ vector within current time window, $t=1, \ldots, L, t$ is time index, numerating vectors. Our purpose is investigating clustering properties of clouds of $4 \mathrm{D}$ vectors $\vec{\xi}^{(t)}$ with each 1-year time window. In particular, we are interesting what is the "best" number of clusters.

Before making cluster procedure 2 preliminary operations were performed within each time window. The $1^{\text {st }}$ operation is normalizing and winsorizing [19] of each scalar component $\xi_{k}^{(t)}$ of vectors $\vec{\xi}^{(t)}$ within each time window. Here $k=1, \cdots, 4$ is the index numerating scalar components of the vector $\vec{\xi}^{(t)}$. Let

$$
\bar{\xi}_{k}=\frac{1}{L} \sum_{t=1}^{L} \xi_{k}^{(t)}, \quad \sigma_{k}^{2}=\frac{1}{(L-1)} \sum_{t=1}^{L}\left(\xi_{k}^{(t)}-\bar{\xi}_{k}\right)^{2}
$$

be sample estimates of mean values and variance of scalar components of the $4 \mathrm{D}$ vector $\vec{\xi}^{(t)}$. Let's perform iterations which consist in coming to values $\xi_{k}^{(t)}=\left(\xi_{k}^{(t)}-\bar{\xi}_{k}\right) / \sigma_{k}$ and clipping values $\xi_{k}^{(t)}$ exceeding over and under thresholds $\pm 4 \sigma_{k}$. These iterations are stopped when the values $\bar{\xi}_{k}$ and $\sigma_{k}$ became stable and equal to the following values: $\bar{\xi}_{k}=0, \alpha_{k}^{2}=1$

The $2^{\text {nd }}$ preliminary operation is coming from $4 \mathrm{D}$ vectors $\vec{\xi}^{(t)}$ to 3D vectors $\vec{\eta}^{(t)}$ of first principal components by projecting vectors $\vec{\zeta}^{(t)}$ on eigenvectors of covariance matrix corresponding to its 3 first maximum eigenvalues.

After these 2 preliminary operations at each current time window we have a cloud consisting of $L 3 \mathrm{D}$ vectors $\vec{\eta}^{(t)}$. Let's split some cloud into given number $q$ of clusters using standard $k$-means cluster procedure [20]. Let $\Gamma_{r}, r=1, \ldots, q$ be clusters, $\vec{z}^{(r)}=\sum_{\vec{\eta} \in \Gamma_{r}} \vec{\eta}^{(t)} / n_{r}-$ mean vector of the cluster $\Gamma_{r}, n_{r}$ be a number of vectors $\vec{\eta}^{(t)}$ within cluster $\Gamma_{r}, \sum_{r=1}^{q} n_{r}=L . K$-means procedure minimizes sum $S\left(\vec{z}^{(1)}, \ldots, \vec{z}^{r=1}\right)=\sum_{r=1}^{q} \sum_{i \in \in \Gamma_{r}}\left|\vec{\eta}^{(t)}-\vec{z}^{(r)}\right|^{2}$ with respect to positions of clusters' centers $\vec{z}^{(r)}$. Let $J(q)=\min _{\vec{z}^{(1)}, \ldots, \vec{z}^{(q)}} S\left(\vec{z}^{(1)}, \ldots, \vec{z}^{(q)}\right)$. We try probe number of clusters within range $2 \leq q \leq 40$. The problem of selecting the best number of clusters $q$ was solved from maximum of pseudo-F-statistics [21]:

$$
\operatorname{PFS}(q)=\sigma_{1}^{2}(q) / \sigma_{0}^{2}(q) \rightarrow \max _{2 \leq q \leq 40},
$$

where $\sigma_{0}^{2}(q)=J(q) /(L-q)$,

$$
\sigma_{1}^{2}(q)=\sum_{r=1}^{q} v_{r} \cdot\left|\vec{z}^{(r)}-\vec{z}^{(0)}\right|^{2} /(q-1), v_{r}=n_{r} / L
$$



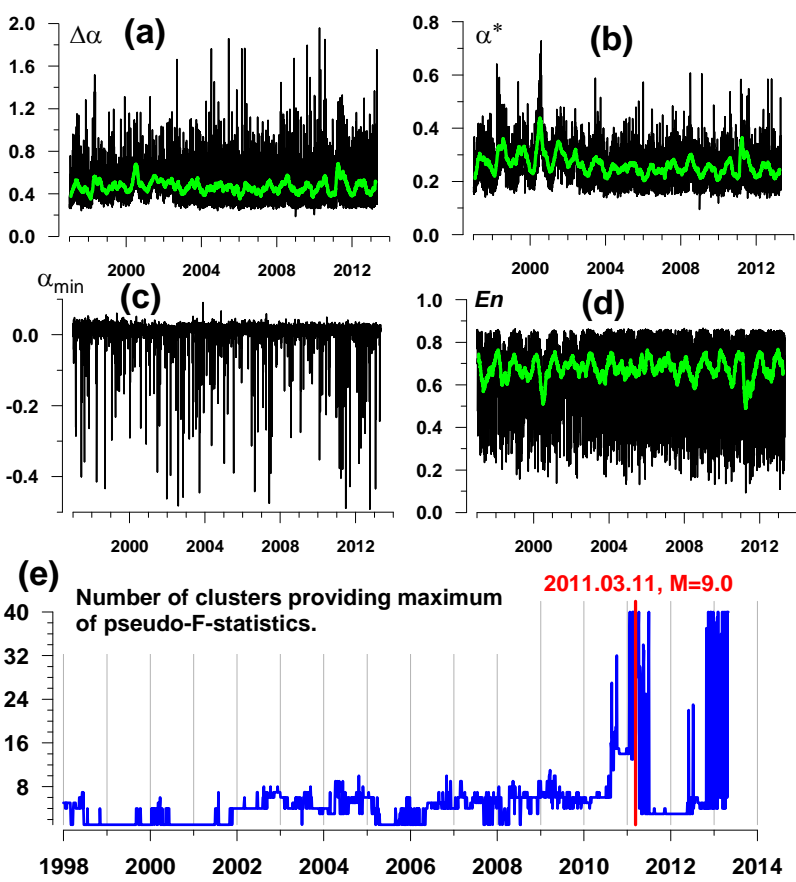

Right-hand end of 365 days moving time window with mutual shift 3 days

Figure 5. (a)-(d) - median values of 4 daily parameters of seismic noise $\Delta \alpha, \alpha^{*}, \alpha_{\min }$-multi-fractal singularity spectra parameters; $\boldsymbol{E n}$-minimum normalized entropy of squared orthogonal wavelet coefficients, green lines-running average within 57 days moving time window. (e) — result of clustering of 3 first principal components of medians of 4 daily seismic noise parameters (a)-(d) within moving windows of the length 365 days with mutual shift 3 days.

$\vec{z}^{(0)}=\sum^{L} \vec{\eta}^{(t)} / L$ - mean vector of the whole cloud of principłal components $\bar{\eta}^{(t)}$.

The $P F S \rightarrow$ max rule is not working if we try to distinguish cases $q^{*}=1$ and $q^{*}=1$ because the value $\sigma_{1}^{2}(q)$ is not defined for $q=1$. These cases could be distinguished by existing of break point of the monotonous function $J(q)$ at the argument $q=2$ [11]. Let $\delta(q)$ be the deflection of the dependence of $\ln \left(\sigma_{0}^{2}(q)\right)$ on $\ln (q)$ from linear approximation:

$\ln \left(\sigma_{0}^{2}(q)\right)=a \cdot \ln (q)+b+\delta(q)$, where coefficients ( $a$, $b$ ) are found by least squares: $\sum_{q=1}^{40} \delta^{2}(q) \rightarrow \min$. The final rule for selecting $q^{*}$ is the following.

Let $q_{0}=\underset{2 \leq q \leq 40}{\arg \max } \operatorname{PFS}(q)$. If $q_{0}>2$ then $q^{*}=q_{0}$. Else if $\delta(1) / \max _{2 \leq q \leq 40} \delta(q) \leq 1$ then $q^{*}=1$ else $q^{*}=2$.

Graphic at Figure 5(e) presents evolution of the estimates of the best number of clusters $q^{*}$ in dependence on the right-hand end of moving time window of the length 1 year. This plot contains the most intrigue characteristics of the data: now we observe the same unstable behavior of $q^{*}$ which was observed before 2011.03.11 and during some time immediately after Tohoku mega-earthquake.
A question arises: does the Figure 5(e) mean that the next mega-earthquake is already prepared and waits for its trigger?

\section{CONCLUSION}

The averaged maps of singularity spectra support width and minimum normalized entropy of squared orthogonal wavelet coefficients of low-frequency seismic noise could be regarded as a new tool of dynamic estimate of seismic danger. These maps give a possibility to inspect the origin and evolution of the SSD — "spots of seismic danger”. Analysis of seismic noise at Japan islands from broad-band seismic network F-net gave a possibility for prediction of Tohoku mega-earthquake 2011.03.11, which was published in advance of the event. According to the analysis of seismic noise (correlation $\rho^{2}$ between 2 multi-fractal parameters and the "best" number $q^{*}$ of clusters for annual clouds of properties of median values of 4 daily statistics) after 2011.03.11 the next mega-earthquake with magnitude near 9 could occur at the region of Nankai Trough during period 2013-2014.

\section{ACKNOWLEDGEMENTS}

This work was supported by the Russian Foundation for Basic Research (project No. 12-05-00146) and Russian Ministry of Science (project No. 11.519.11.5024). The author is grateful to National Institute for Earth Science and Disaster Prevention (NIED), Japan, for providing free access to the source of broadband seismic noise waveforms registered at the F-net stations.

\section{REFERENCES}

[1] Rikitake, T. (1999) Probability of a great earthquake to recur in the Tokai district, Japan: Reevaluation based on newly-developed paleoseismology, plate tectonics, tsunami study, micro-seismicity and geodetic measurements. Earth, Planets and Space, 51, 147-157.

[2] Mogi, K. (2004) Two grave issues concerning the expected Tokai Earthquake. Earth, Planets and Space, 56, li-lxvi.

[3] Simons, M., Minson, S.E., Sladen, A., Ortega, F., Jiang, J., Owen, S.E., Meng, L., Ampuero, J.-P., Wei, S., Chu, R., Helmberger, D.V., Kanamori, H., Hetland, E., Moore, A.W. and Webb, F.H. (2011) The 2011 magnitude 9.0 Tohoku-Oki earthquake: Mosaicking the megathrust from seconds to centuries. Science, 332, 911. doi:10.1126/science.332.6032.911

[4] Kagan Y.Y. and Jackson D.D. (2013) Tohoku earthquake: A Surprise? Bulletin of the Seismological Society of America, 103, 1181-1194. doi:10.1785/0120120110

[5] Kobayashi N. and Nishida, K. (1998) Continuous excitation of planetary free oscillations by atmospheric disturbances. Nature, 395, 357-360. doi:10.1038/26427

[6] Tanimoto, T. (2005) The oceanic excitation hypothesis for 
the continuous oscillations of the Earth. Geophysical Journal International, 160, 276-288.

doi:10.1111/j.1365-246X.2004.02484.X

[7] Rhie, J. and Romanowicz, B. (2004) Excitation of Earth's continuous free oscillations by atmosphere-ocean-seafloor coupling. Nature, 431, 552-554. doi:10.1038/nature02942

[8] Lyubushin, A.A. (2009) Synchronization trends and rhythms of multifractal parameters of the field of lowfrequency microseisms. Izvestiya, Physics of the Solid Earth, 45, 381-394. doi:10.1134/S1069351309050024

[9] Lyubushin, A.A. (2010) The statistics of the time segments of low-frequency microseisms: Trends and synchronization. Izvestiya, Physics of the Solid Earth, 46, 544-554. doi:10.1134/S1069351310060091

[10] Lyubushin, A. (2010) Multifractal parameters of lowfrequency microseisms. In: de Rubeis, V., et al., Eds., Synchronization and Triggering: From Fracture to Earthquake Processes. GeoPlanet: Earth and Planetary Sciences 1, Springer-Verlag, Berlin Heidelberg, 2010, Chapter 15, 253-272. doi:10.1007/978-3-642-12300-9_15

[11] Lyubushin, A.A. (2011) Cluster analysis of low-frequency microseismic noise. Izvestiya, Physics of the Solid Earth, 47, 488-495. doi:10.1134/S1069351311040057

[12] Lyubushin, A.A. (2011) Seismic catastrophe in Japan on March 11, 2011: Long-term prediction on the basis of low-frequency microseisms. Izvestiya, Atmospheric and Oceanic Physics, 46, 904-921. doi:10.1134/S0001433811080056

[13] Lyubushin, A. (2012) Prognostic properties of low-fre- quency seismic noise. Natural Science, 4, 659-666. doi:10.4236/ns.2012.428087

[14] Lyubushin, A.A. (2013) Mapping the Properties of LowFrequency Microseisms for Seismic Hazard Assessment. Izvestiya, Physics of the Solid Earth, 49, 9-18. doi:10.1134/S1069351313010084

[15] Lyubushin, A.A. (2013) Spots of seismic danger extracted by properties of low-frequency seismic noise. Geophysical Research Abstracts, Vol. 15, 2013, 1 Pages.

[16] Feder, J. (1988) Fractals. Plenum Press, New York, London.

[17] Kantelhardt, J.W., Zschiegner, S.A., Konscienly-Bunde, E., Havlin, S., Bunde, A. and Stanley, H.E. (2002) Multifractal detrended fluctuation analysis of nonstationary time series. Physica A, 316, 87-114. doi:10.1016/S0378-4371(02)01383-3

[18] Mallat, S. (1998) A wavelet tour of signal processing. Academic Press, San Diego, London, Boston, Sydney, Tokyo, Toronto.

[19] Huber, P.J. and Ronchetti, E.M. (2009) Robust statistics, 2nd Edition, John Wiley \& Sons, Inc., Hoboken, 354 Pages. doi:10.1002/9780470434697

[20] Duda, R.O., Hart, P.E. and Stork, D.G. (2000) Pattern Classification. Wiley-Interscience Publication, Hoboken, 680 Pages.

[21] Vogel, M.A. and Wong, A.K.C. (1979) PFS Clustering method. IEEE Transactions on Pattern Analysis and Machine Intelligence, 1, 237-245. doi:10.1109/TPAMI.1979.4766919 